\title{
Clinical outcomes of intracranial solitary fibrous tumor and hemangiopericytoma: analysis according to the 2016 WHO classification of central nervous system tumors
}

\author{
*Byung Sup Kim, MD, ${ }^{1}$ Yuil Kim, MD, PhD, ${ }^{2}$ Doo-Sik Kong, MD, PhD, ${ }^{3}$ Do-Hyun Nam, MD, PhD, ${ }^{3}$ \\ Jung-II Lee, MD, PhD, ${ }^{3}$ Yeon-Lim Suh, MD, PhD, ${ }^{4}$ and Ho Jun Seol, MD, PhD ${ }^{3}$ \\ 1Department of Neurosurgery, Kosin University Gospel Hospital, Kosin University College of Medicine, Busan; ${ }^{2}$ Department \\ of Pathology, Seoul St. Mary's Hospital, The Catholic University of Korea, Seoul; and Departments of ${ }^{3}$ Neurosurgery and \\ ${ }^{4}$ Pathology, Samsung Medical Center, Sungkyunkwan University School of Medicine, Seoul, Republic of Korea
}

OBJECTIVE The authors conducted this retrospective study to investigate the clinical outcomes of intracranial solitary fibrous tumor (SFT) and hemangiopericytoma (HPC), defined according to the 2016 WHO classification of central nervous system (CNS) tumors.

METHODS Histopathologically proven intracranial SFT and HPC cases treated in the period from June 1996 to September 2014 were retrospectively reviewed and analyzed. Two neuropathologists reviewed pathological slides and regraded the specimens according to the 2016 WHO classification. Factors associated with progression-free survival (PFS) and overall survival (OS) were statistically evaluated with uni- and multivariate analyses.

RESULTS The records of 47 patients-10 with SFT, 33 with HPC, and 4 with anaplastic HPC - were reviewed. A malignant transition from conventional SFT to WHO grade III SFT/HPC was observed in 2 cases, and 13 HPC cases were assigned grade III SFT/HPC. Mean and median follow-ups were 114.6 and 94.7 months, respectively (range 7.1-366.7 months). Gross-total resection (GTR) was significantly associated with longer PFS and OS ( $p=0.012$ for both), and adjuvant radiation therapy versus no such therapy led to significantly longer PFS $(p=0.018)$. Extracranial metastases to the liver, bone, lung, spine, and kidney occurred in 10 patients (21.3\%). Grade III SFT/HPC was strongly correlated with the development of extracranial metastases $(p=0.031)$.

CONCLUSIONS The 2016 WHO classification of CNS tumors reflected the different types of pathological malignant progression and clinical outcomes better than prior classifications. Gross-total resection should be the primary treatment goal in patients with SFT/HPC, regardless of the pathological grade, and radiation can be administered as adjuvant therapy for patients with SFT/HPC that shows an aggressive phenotype or that is not treated with GTR.

https://thejns.org/doi/abs/10.3171/2017.7.JNS171226

KEY WORDS solitary fibrous tumor; hemangiopericytoma; pathological grade; gross-total resection; extracranial metastasis; oncology

$\mathrm{I}$ NTRACRANIAL solitary fibrous tumor (SFT) is a rare mesenchymal neoplasm first described by Carneiro et al. ${ }^{3}$ It was originally considered a primary localized neoplasm of the visceral pleura but was later found in almost every extrathoracic location. ${ }^{41}$ This tumor is characterized by fibrous features arising predominantly from thick collagen bands. In contrast, intracranial hemangiopericytoma (HPC) is a rare, highly cellular, vascular- ized mesenchymal tumor that develops from malignant transformation of Zimmermann's pericytes surrounding the capillary and postcapillary venules. ${ }^{39}$ It shows more mitosis and reticulin investment of individual cells. Intracranial SFT and HPC have overlapping histological and immunohistochemical characteristics, including a well-defined tumor border, spindle to oval cells, biphasic hypo- and hypercellular areas, well-developed branching

\footnotetext{
ABBREVIATIONS CNS = central nervous system; EBRT = external beam radiation therapy; EMA = epithelial membrane antigen; GKS = Gamma Knife surgery; GTR = gross-total resection; HPC = hemangiopericytoma; OS = overall survival; PFS = progression-free survival; SFT = solitary fibrous tumor; $S T R=$ subtotal resection. SUBMITTED May 18, 2017. ACCEPTED July 14, 2017.

INCLUDE WHEN CITING Published online January 26, 2018; DOI: 10.3171/2017.7.JNS171226.

* Drs. B. S. Kim and Y. Kim contributed equally to this work and share first authorship. Drs. Suh and Seol contributed equally to this work and share senior authorship.
} 
vasculature, frequent expression of CD34, and negativity for epithelial membrane antigen (EMA). ${ }^{11}$ No clinical or radiological features accurately distinguish SFT from HPC, and in some cases, it is difficult to discriminate SFT from HPC even based on histological and immunohistochemical features. ${ }^{2}$ Nonetheless, intracranial SFT and HPC were declared separate entities with different prognoses. ${ }^{15,24,30}$ Recently, whole-exome sequencing revealed a unique NAB2-STAT6 fusion in SFTs. ${ }^{6}$ A validation study on meningeal tumors showed that the same fusion occurred in SFT and HPC and suggested combining intracranial SFT and HPC into a single disease entity. ${ }^{36}$ For this reason, the $2016 \mathrm{WHO}$ classification of central nervous system (CNS) tumors included the combined term "SFT/HPC" to describe such lesions, and 3 grades were assigned to the entity of SFT/HPC. ${ }^{25}$ The changes in the pathological classification may represent the different clinical prognoses and the requirement for different treatment strategies. Although most studies have recommended a multimodal treatment strategy, very little of the published literature has reported long-term follow-ups, and only a few studies have suggested that the 2016 WHO classification reflects patient prognosis better than prior pathological classifications. When considering observation or additional treatment after surgery for intracranial SFT/HPC, it is important to understand the clinical outcomes of this entity in order to improve patient outcomes. The purpose of the present study was to investigate the clinicopathological factors of intracranial SFT/HPC that affect clinical progression and patient survival and to suggest an appropriate treatment strategy based on the 2016 WHO classification. Here, we analyzed the clinical outcomes of 47 patients with SFT/HPC when considering the changes in classification.

\section{Methods}

This study was approved by the institutional review board at our institute and is in accordance with the Declaration of Helsinki. We collected from the pathology database at our hospital those cases that had been histologically diagnosed as intracranial SFT, HPC, and anaplastic HPC. Medical and radiological records for the patients were retrospectively reviewed. All patients had initially undergone resection. Two neuropathologists performed the pathological review. Information on age, sex, tumor location, tumor size, extent of resection, pathology, adjuvant radiotherapy, tumor recurrence or metastasis, and patient survival was collected. Tumor size was obtained by measuring the maximal diameter on the preoperative MR images. The neuroimaging evaluation of all patients had been conducted preoperatively as well as within 3 months of surgery. The extent of resection was classified as grosstotal resection (GTR) or subtotal resection (STR) based on the postoperative MRI study. Gross-total resection was defined as Simpson grade I or II removal, whereas STR was defined as Simpson grade III removal. Adjuvant radiotherapy, such as external beam radiation therapy (EBRT) or Gamma Knife surgery (GKS), was suggested for tumors that manifested malignant pathological features or that were not treated with GTR. Progression was defined as the reappearance of tumor within the cranial cavity or an increase in the size of residual tumor. Metastasis was defined as the presence of HPC tissue in extracranial locations.

\section{Pathology}

The typical SFT shows a patternless architecture characterized by a combination of alternating hypocellular and hypercellular areas separated from each other by thick bands of hyalinized, sometimes keloidal or amianthoidlike collagen and thin-walled staghorn vessels. The typical HPC is characterized by highly cellular, less collagenous tumors with plump cells and thin-walled, branching HPClike (staghorn) vessels. In a 3-tiered system, classic SFT was histologically considered benign at grade I, whereas HPC was considered malignant. Hemangiopericytomas were subclassified as grade II or grade III (anaplastic) depending on the mitotic count $(<5$ vs $\geq 5$ mitoses per 10 hpf) and/or necrosis plus at least 2 of the following: hemorrhage, moderate to high nuclear atypia, or cellularity. ${ }^{24,28}$ All specimens were regraded by the 2 neuropathologists (Y.K. and Y.L.S.) according to the 2016 WHO classification. There was no disagreement on the pathological diagnosis between the 2 neuropathologists. They doublechecked the diagnoses of the included cases. At the time of diagnosis, cases that had been hard to differentiate from meningioma had a differential diagnosis via reticulin and immunohistochemical staining using EMA, claudin-1, claudin-11, CD34, Bcl-2, CD99, and electron microscopy study. Meningioma was reactive to at least 1 of 3 markers (EMA, claudin-1, or claudin-11). In cases with overlapping histopathological and immunohistochemical features of meningioma and SFT/HPC, meningioma could be excluded based on diagnostic ultrastructural features, such as complex interdigitating cellular processes and desmosomal intercellular junctions. The 2016 WHO classification assigns 3 grades within the entity of SFT/HPC: ${ }^{25}$ grade I is defined as the tumors previously diagnosed as SFT (Fig. 1A); grade II, as the tumors previously diagnosed as HPC in the CNS (Fig. 1B); and grade III, as the tumors previously diagnosed as anaplastic HPC on the basis of $\geq 5$ mitoses per $10 \mathrm{hpf}$ (Fig. 1C). Solitary fibrous tumor with malignant features such as high mitotic activity ( $\geq$ 5 mitoses per $10 \mathrm{hpf}$ ) was assigned a WHO grade III. A comparison between old and new WHO classifications is featured in Table 1. According to the original diagnoses in the chart review, 10 patients had SFT, 33 had HPC, and 4 had anaplastic HPC. Twenty-two cases diagnosed as HPC before 2007 had not been graded, 11 of which were reclassified as HPC grade III based on the 2016 WHO classification of CNS tumors.

\section{Statistical Analysis}

Progression-free survival (PFS) was calculated as the time from the date of initial surgery following a pathological diagnosis of SFT or HPC to the date of documented tumor recurrence or further growth of residual tumor. Overall survival (OS) was calculated as the time from the initial surgery to death or the last-known date of posttreatment follow-up. Analyses for progression and 


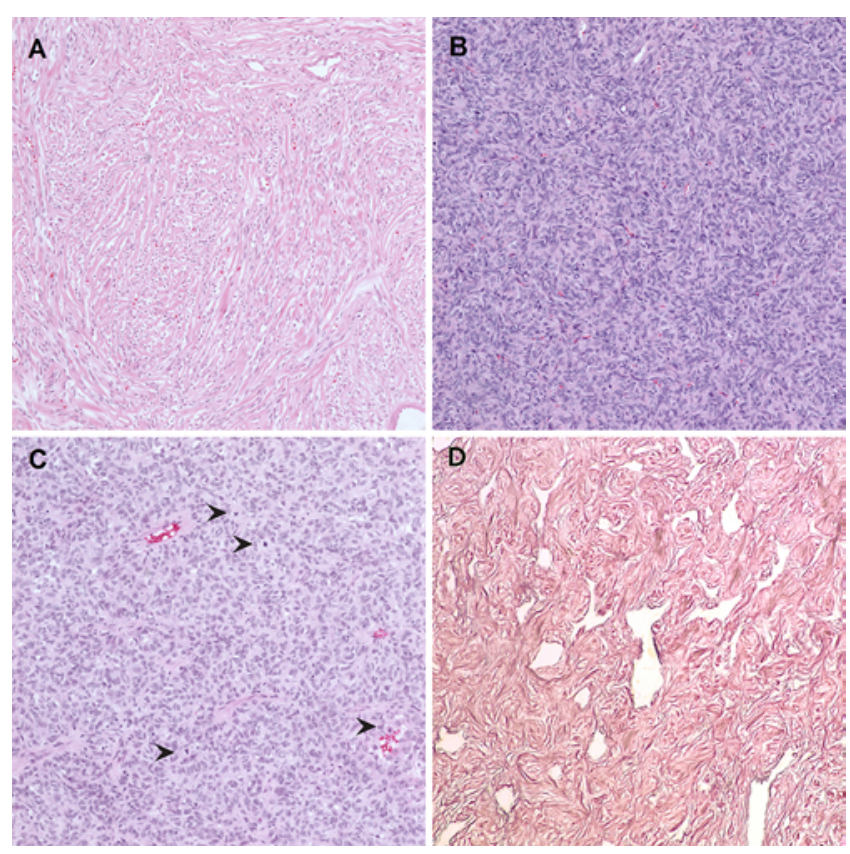

FIG. 1. Typical microscopic features of SFT/HPC according to the 2016 WHO classification of CNS tumors. A: Grade I SFT/HPC. Spindle to oval cells are interspersed with prominent bands of collagen. B: Grade II SFT/HPC. Spindle to oval cells are more densely distributed without predominance of collagen bundles. By definition, mitosis is $<5$ per 10 hpf. C: Grade III SFT/HPC. An example with a high mitotic rate (arrowheads) and necrosis (not shown). D: Reticulin stain shows the dilated staghorn vascular pattern of HPC. $\mathrm{H} \& \mathrm{E}(\mathrm{A}-\mathrm{C})$, original magnification $\times 200$ (A-D). Figure is available in color online only.

survival were based on the primary intervention that a patient received. The factors associated with PFS and OS were statistically evaluated with uni- and multivariate analyses. Univariate survival analysis was performed using the Kaplan-Meier method to evaluate prognostic factors. Significance was determined using the log-rank test, with a $\mathrm{p}$ value considered significant at the $5 \%$ level (that is, $\mathrm{p}<0.05$ ). A multivariate Cox regression analysis was then performed to determine the independent impact of factors found to be significant in the univariate analysis.
All statistical analyses were performed using IBM SPSS Statistics 23.0 (IBM Corp.).

\section{Results \\ Patient Characteristics}

Forty-seven patients with primary intracranial SFT or HPC were treated in our department in the period from June 1996 to September 2014 (Table 2). There were 30 male $(63.8 \%)$ and 17 female $(36.2 \%)$ patients, with a median age of 38 years (range 22-77 years) at initial diagnosis. Thirty-nine tumors $(83.0 \%)$ were in supratentorial locations and $8(17.0 \%)$ in infratentorial locations. The mean and median follow-up periods were 114.6 and 94.7 months, respectively (range 7.1-366.7 months). At the first visit, the median maximal tumor diameter measured on brain MR images was $6.0 \mathrm{~cm}$ (range 2.3-9.0 cm). Twenty-three patients $(48.9 \%)$ had undergone preoperative embolization. Ten patients $(21.3 \%)$ had died by the time of the retrospective analysis. The mean survival time across all patients was 246.7 months (95\% CI 188.1-305.4 months) after the date of diagnosis, with 1-, 5-, and 10-year survival rates of $97.8 \%, 92.7 \%$, and $69.5 \%$, respectively. Twenty-seven patients $(57.4 \%)$ experienced at least 1 progression or recurrence. The mean time to progression or recurrence was 102.0 months (95\% CI 74.4-129.6 months) after the date of diagnosis, with 1-, 5-, and 10-year PFS rates of $87.1 \%$, $56.0 \%$, and $34.6 \%$, respectively. Ten patients $(21.3 \%)$ developed extracranial metastases. The mean time to the development of extracranial metastases was 226.8 months (95\% CI 168.3-285.4 months). The extracranial metastasis-free survival rates at 1,5 , and 10 years were $97.4 \%$, $94.5 \%$, and $81.1 \%$, respectively. The sites of extracranial metastases were the liver ( 7 patients), bones ( 7 patients), lung (5 patients), spine (5 patients), and kidney ( 2 patients).

\section{Univariate and Multivariate Analyses of Factors Related to PFS and OS}

We evaluated the effect of prognostic values on PFS and OS for variables such as age, sex, tumor size, tumor location, extent of resection, adjuvant radiation, and pathology. Univariate analyses showed that the extent of resection and adjuvant radiation were significantly associated

\section{TABLE 1. Comparison between old and new WHO criteria for CNS tumors}

\begin{tabular}{|c|c|}
\hline Prior (2007) WHO Classification* & New (2016) WHO Classification† \\
\hline $\begin{array}{l}\text { SFT \& HPC represent separate entities. } \\
\text { SFT defined as benign mesenchymal tumor consisting } \\
\text { of patternless architecture or short fascicular pattern } \\
\text { w/ alternating hypocellular \& hypercellular areas w/ } \\
\text { thick bands of collagen. } \\
\text { HPC defined as highly cellular mesenchymal tumor w/ } \\
\text { dilated staghorn-type vasculatures \& classified into } \\
\text { HPC WHO grade II \& anaplastic HPC WHO grade } \\
\text { III w/ high mitotic activity ( }>5 \text { mitoses } / 10 \text { hpf) \&/or } \\
\text { necrosis. }\end{array}$ & $\begin{array}{l}\text { Restructuring of SFT \& HPC as } 1 \text { entity (SFT/HPC) w/ histological spectrum of tumors \& } \\
\text { assigning } 3 \text { grades w/in this single entity. } \\
\text { Grade I: most often corresponds to highly collagenous, relatively low-cellularity spindle- } \\
\text { cell lesion previously diagnosed as SFT. } \\
\text { Grade II: typically corresponds to more cellular, less collagenous tumor w/ plump cells \& } \\
\text { staghorn vasculature that was previously diagnosed as HPC in the CNS. } \\
\text { Grade III: corresponds to what was termed "anaplastic HPC" in the past; diagnosed on } \\
\text { the basis of } \geq 5 \text { mitoses/ } 10 \text { hpf. } \\
\text { Some tumors w/ histological appearance more similar to traditional SFT can also display } \\
\text { malignant features \& be assigned WHO grade III, using a cutoff of } \geq 5 \text { mitoses } / 10 \mathrm{hpf.}\end{array}$ \\
\hline
\end{tabular}

* Louis et al., 2007.

$\dagger$ Louis et al., 2016. 
TABLE 2. Summary of characteristics in 47 patients with SFT/HPC

\begin{tabular}{|c|c|}
\hline Characteristic & Value \\
\hline No. of patients & 47 \\
\hline \multicolumn{2}{|l|}{ Age in yrs (no. of patients [\%]) } \\
\hline 20 to $<40$ & $27(57.4)$ \\
\hline 40 to $<60$ & $17(36.2)$ \\
\hline$\geq 60$ & $3(6.4)$ \\
\hline $\operatorname{Sex}(M / F)$ & $30: 17$ \\
\hline Median FU in mos (range) & $94.7(7.1-366.7)$ \\
\hline Mean FU in mos (range) & $114.6(7.1-366.7)$ \\
\hline \multicolumn{2}{|l|}{ Tumor location (no. [\%]) } \\
\hline Supratentorial & $39(83.0)$ \\
\hline Infratentorial & $8(17.0)$ \\
\hline \multicolumn{2}{|c|}{ Tumor size in cm (no. of patients [\%]) } \\
\hline$<3.0$ & $3(6.4)$ \\
\hline 3.0 to $<6.0$ & $19(40.4)$ \\
\hline$\geq 6.0$ & $25(53.2)$ \\
\hline \multicolumn{2}{|l|}{ Extent of resection (no. [\%]) } \\
\hline GTR & $33(70.2)$ \\
\hline STR & $14(29.8)$ \\
\hline Postop radiotherapy (no. [\%]) & $17(36.2)$ \\
\hline Postop GKS (no. [\%]) & $2(4.3)$ \\
\hline \multicolumn{2}{|l|}{ Prior pathology (no. [\%]) } \\
\hline SFT & $10(21.3)$ \\
\hline HPC & $33(70.2)$ \\
\hline Anaplastic HPC & $4(8.5)$ \\
\hline \multicolumn{2}{|c|}{ Pathology per 2016 WHO scheme (no. [\%]) } \\
\hline SFT/HPC grade I & $8(17.0)$ \\
\hline SFT/HPC grade II & $20(42.6)$ \\
\hline SFT/HPC grade III & $19(40.4)$ \\
\hline \multicolumn{2}{|l|}{ Recurrence (no. [\%]) } \\
\hline Yes & $27(57.4)$ \\
\hline No & $20(42.6)$ \\
\hline \multicolumn{2}{|l|}{ Extracranial metastasis (no. [\%]) } \\
\hline Yes & $10(21.3)$ \\
\hline No & $37(76.7)$ \\
\hline
\end{tabular}

FU = follow-up.

with tumor recurrence $(\mathrm{p}<0.05)$, whereas age, sex, tumor location, tumor size, and pathology were not. Both GTR and adjuvant radiation, as compared with STR and the absence of adjuvant radiation, were significantly associated with longer PFS ( $p<0.05)$. Age, sex, and extent of resection were also positively correlated with OS $(\mathrm{p}<0.05)$. An age over 60 years, male sex, and GTR were associated with significantly better OS $(\mathrm{p}<0.05)$ than were an age under 60 years, female sex, and STR. The factors of sex, extent of resection, adjuvant radiation, and pathology were included in the Cox regression analyses. (Because some of the literature has reported pathology as a significant prognostic factor and given the possibility of selection bias, we included pathology in our regression analysis.) The results revealed that GTR remained significantly and positively correlated with PFS and OS ( $\mathrm{p}<0.05)$. Adjuvant radiation was significantly positively correlated with PFS $(\mathrm{p}=$ 0.033; Table 3).

\section{Pathology}

Applying the 2016 WHO classification, we reclassified conventional SFT as WHO grade III SFT/HPC in 2 cases (Fig. 2), and 13 original HPC cases were relabeled as WHO grade III SFT/HPC (Fig. 3). All 4 anaplastic HPCs were corroborated as WHO grade III SFT/HPC. Therefore, according to the 2016 WHO classification, there were 8 (17.0\%) grade I SFT/HPC, $20(42.6 \%)$ grade II SFT/HPC, and 19 (40.4\%) grade III SFT/HPC. In 1 of the 2 cases that had been classified as SFT in the previous pathological classification and relabeled as WHO grade III SFT/HPC, recurrence and extracranial metastases occurred, and the patient finally died as a result of the extracranial metastases. Among the 13 cases that had been classified as HPC in the previous pathological classification and relabeled as WHO grade III SFT/HPC, 12 patients $(92.3 \%)$ experienced recurrence, 7 patients $(53.8 \%)$ had extracranial metastases, and 6 patients $(46.2 \%)$ died. The grade III SFT/HPC group that had been regraded from conventional SFT or HPC showed higher recurrence rates, more frequent extracranial metastases, and higher mortality rates (Table 4), and the 2016 WHO classification reflected the malignant pathological potential better than the 2007 pathological classification. When considering PFS and OS according to the 2007 WHO pathological classification, the SFT group demonstrated significantly superior PFS than the HPC and anaplastic HPC group (137.2 vs 87.7 months, $p=0.039$; Fig. 4A), whereas the anaplastic HPC group had significantly worse OS than the SFT and HPC group (51.8 vs 251.7 months, $\mathrm{p}=0.014$; Fig. 4B). When analyzing PFS and OS according to the 2016 WHO classification, the grade I SFT/HPC group demonstrated significantly superior PFS than the grade II and III SFT/HPC group (146.2 vs 89.7 months, $\mathrm{p}=0.043$; Fig. $4 \mathrm{C})$. Fourteen (51.9\%) of 27 recurrences were classified as grade III SFT/HPC. The grade III SFT/HPC group had significantly worse OS than the grade I and II SFT/HPC group (194.8 vs 220.8 months, $\mathrm{p}=0.048$; Fig. 4D). There was no difference in prognosis for PFS and OS between the prior pathological classification and the 2016 WHO classification. Eight (80\%) of 10 patients with extracranial metastases showed grade III SFT/HPC pathology and the other 2 patients (20\%) showed grade II SFT/HPC pathology. Grade III SFT/HPC was strongly correlated with the development of extracranial metastases $(p=0.031$; Fig. 5A). The mean time to the development of extracranial metastases was 208.7 months (95\% CI 185.2-232.1 months) in the grade I and II SFT/HPC group and 184.2 months (95\% CI 116.6-251.7 months) in the grade III SFT/ HPC group. Five (50\%) of the 10 patients with extracranial metastases had died by the time of our analysis, and all 5 showed grade III SFT/HPC pathology.

\section{Extent of Resection}

Gross-total resection was achieved in 33 patients (70.2\%) and STR in 14 patients (29.8\%). After GTR, 13 


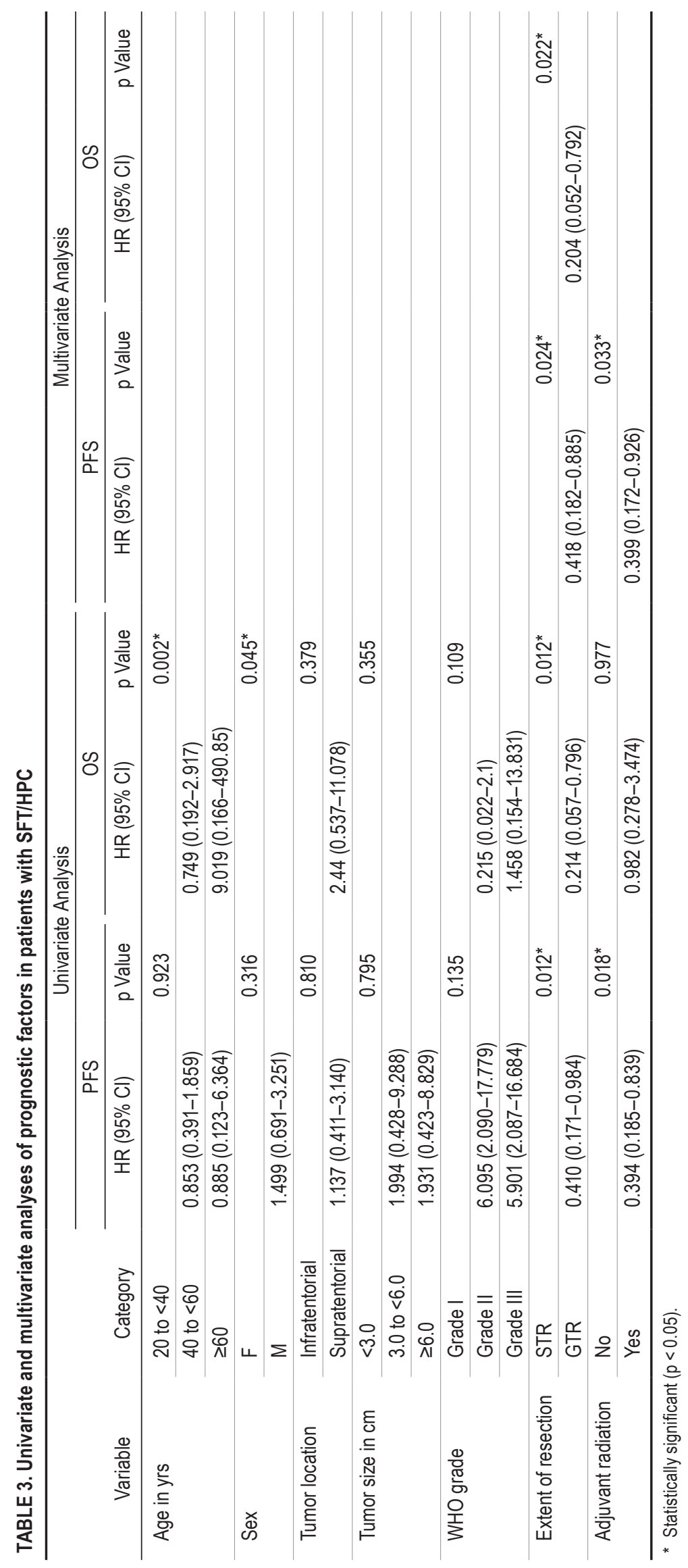




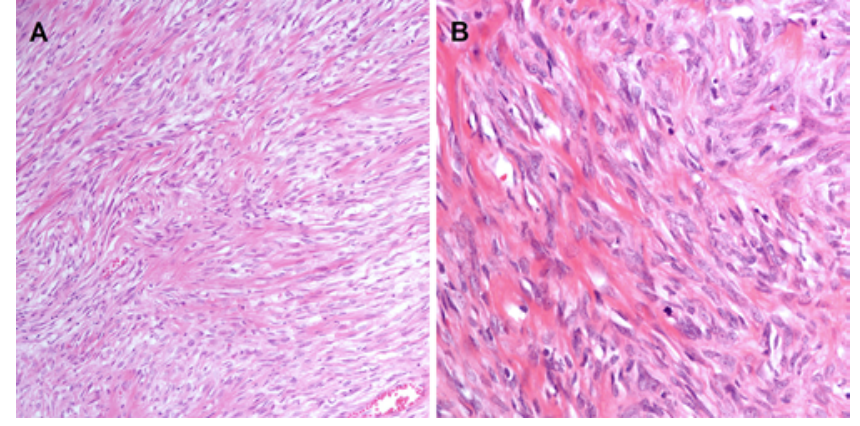

FIG. 2. Representative example reclassified from conventional SFT to grade III SFT/HPC according to the 2016 WHO classification. Histologically, this tumor consists of fascicles of spindle cells with prominent eosinophilic bands of collagen $(A)$ and frequent mitosis $(B)$. The patient was initially treated with GKS after STR, and the tumor progressed intracranially at 61 months after treatment. Extracranial metastases to the liver and lung occurred at 88.1 months, and the patient died 106.2 months after initial diagnosis. $H \& E$, original magnification $\times 200(A)$ and $\times 400$ (B). Figure is available in color online only.

(39.4\%) of 33 patients received adjuvant EBRT and 1 (3.0\%) of 33 underwent GKS. Five patients $(35.7 \%)$ who had undergone STR were further treated with adjuvant radiation, including $4(28.6 \%)$ who received EBRT and 1 (7.1\%) who underwent GKS. Three patients $(9.1 \%)$ who had undergone GTR and 7 (50.0\%) who had undergone STR had died by the time of our analysis (Table 5). The mean OS time was 293.9 months (95\% CI 219.3-368.5 months) in the GTR group and 151.4 months (95\% CI 105.5-197.3 months) in the STR group ( $\mathrm{p}=0.012$; Fig. 6B). Fifteen patients $(45.5 \%)$ who had undergone GTR and 12 (85.7\%) who had undergone STR experienced recurrence. The mean time to progression or recurrence was 127.6 months (95\% CI 91.5-163.7 months) in the GTR group and 56.3 months (95\% CI 35.5-77.1 months) in the STR group ( $\mathrm{p}=$ 0.012; Fig. 6A). Expected mean PFS in the GTR group was 105.6 months (95\% CI 56.6-154.6 months). Actuarial PFS rates at 1 and 5 years were $78.9 \%$ and $41.6 \%$, respectively. Extracranial metastases developed in 4 patients $(12.1 \%)$ who had undergone GTR and in 6 patients $(42.9 \%)$ who had undergone STR. The mean time to the development of extracranial metastases was 270.2 months $(95 \%$ CI $196.3-$ 344.1 months) in the GTR group and 149.4 months (95\% CI 108.2-190.6 months) in the STR group ( $p=0.014$; Fig. 5B).

\section{Multidisciplinary Approach Combining Radical Resection With Adjuvant Radiation Therapy}

Patients undergoing any form of adjuvant radiation treatment, including EBRT and GKS, had longer mean PFS than those who did not undergo such treatment (126.9 vs 74.8 months, $\mathrm{p}=0.018$; Fig. $6 \mathrm{C}$ ). When considering the extent of resection, patients who had undergone GTR alone demonstrated significantly superior OS than those who had undergone STR with or without adjuvant radiation (327.5 vs 151.4 months, $\mathrm{p}=0.048$; Fig. 6D). Patients who had undergone GTR with adjuvant radiation showed a trend toward an increased time to recurrence as compared with those who had undergone GTR alone $(p=0.076)$. We found no significant difference in the time to recurrence

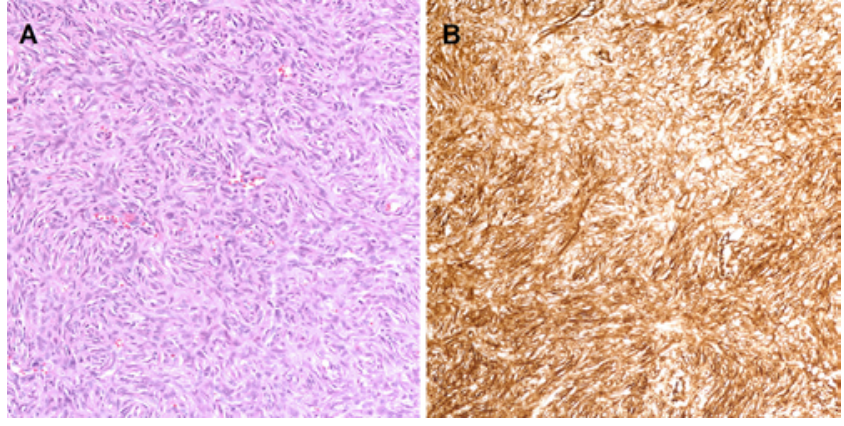

FIG. 3. Representative example reclassified from HPC to grade III SFT/HPC according to the 2016 WHO classification. Histologically, the tumor consists of more cellular, round to oval-shaped cells that are arranged in a short storiform pattern (A). Immunohistochemically, tumor cells are strongly positive for CD34 (B). The patient was initially treated with EBRT after GTR, and the tumor progressed intracranially at 90.4 months after treatment. Extracranial metastases to the spine occurred at 223.3 months, and the patient died 225.7 months after initial diagnosis. $H \& E(A)$, original magnification $\times 200(A)$ and $\times 400(B)$. Figure is available in color online only.

between the patients treated with GTR alone and those treated using STR with or without adjuvant radiation $(\mathrm{p}=$ 0.261 ), between patients treated with GTR alone and those treated with STR plus adjuvant radiation $(\mathrm{p}=0.954)$, or between patients treated with STR plus adjuvant radiation and those treated with STR alone $(\mathrm{p}=0.422)$. Patients who had undergone any form of adjuvant radiation treatment with resection demonstrated worse OS than those who had undergone surgery alone, but the difference was not statistically significant (196.1 vs 257.8 months, respectively, $\mathrm{p}=0.977$ ). In an analysis of the extent of resection, there was a statistical trend toward better OS among the patients who had undergone GTR alone versus those who had undergone STR with adjuvant radiation (332.4 vs 112.2 months, $p=0.076$ ). We found no significant difference in OS between patients treated with GTR plus adjuvant radiation and those treated with GTR alone $(\mathrm{p}=0.562)$ or between patients treated with STR plus adjuvant radiation and those treated with STR alone $(\mathrm{p}=0.854)$. Extracranial metastasis in patients treated using STR with or without adjuvant radiation was more common in those with grade III SFT/HPC than in those with grade II SFT/HPC (62.5\% vs $16.7 \%)$.

\section{Discussion}

Intracranial SFT is generally benign since GTR can be achieved and only $14 \%$ of gross-totally resected SFT cases recur. ${ }^{12}$ In contrast, HPC is considered malignant because of its tendency to recur and develop extracranial metastases despite GTR. ${ }^{10,33,40,42}$ Hemangiopericytoma was subclassified into 2 histological subtypes depending on the mitotic count; that is, HPC was considered a WHO grade II tumor, and anaplastic HPC was considered a grade III tumor. ${ }^{24,28}$ Anaplastic HPC with WHO grade III was defined as the presence of high mitotic activity of more than 5 mitoses per $10 \mathrm{hpf}$ and/or necrosis plus at least 2 of the following: hemorrhage, moderate to high nuclear atypia, or cellularity. Hence, adjuvant radiotherapy for patients diagnosed 
TABLE 4. Comparison between prior pathological classifications and the 2016 WHO classification

\begin{tabular}{|c|c|c|c|c|c|c|c|c|c|}
\hline \multicolumn{5}{|c|}{ Prior (2007) WHO Classification } & \multicolumn{5}{|c|}{2016 WHO Classification } \\
\hline Pathology & $\begin{array}{c}\text { No. of } \\
\text { Patients }\end{array}$ & $\begin{array}{c}\text { No. of } \\
\text { Recurrences (\%) }\end{array}$ & $\begin{array}{l}\text { No. of Extracranial } \\
\text { Metastases (\%) }\end{array}$ & $\begin{array}{c}\text { No. of } \\
\text { Deaths (\%) }\end{array}$ & $\begin{array}{l}\text { Pathology } \\
\text { (SFT/HPC) }\end{array}$ & $\begin{array}{c}\text { No. of } \\
\text { Patients }\end{array}$ & $\begin{array}{c}\text { No. of } \\
\text { Recurrences (\%) }\end{array}$ & $\begin{array}{l}\text { No. of Extracranial } \\
\text { Metastases (\%) }\end{array}$ & $\begin{array}{c}\text { No. of } \\
\text { Deaths (\%) }\end{array}$ \\
\hline \multirow[t]{2}{*}{ SFT } & \multirow[t]{2}{*}{10} & \multirow[t]{2}{*}{$2(20.0)$} & \multirow[t]{2}{*}{$1(10.0)$} & \multirow[t]{2}{*}{$2(20.0)$} & Grade I & 8 & $1(12.5)$ & & $1(12.5)$ \\
\hline & & & & & Grade III & 2 & $1(50.0)$ & $1(50.0)$ & $1(50.0)$ \\
\hline \multirow[t]{2}{*}{ HPC } & \multirow[t]{2}{*}{33} & \multirow[t]{2}{*}{$24(72.7)$} & \multirow[t]{2}{*}{$9(27.2)$} & \multirow[t]{2}{*}{$7(21.2)$} & Grade II & 20 & $12(60.0)$ & $2(10.0)$ & $1(5.0)$ \\
\hline & & & & & Grade III & 13 & $12(92.3)$ & $7(53.8)$ & $6(46.2)$ \\
\hline $\begin{array}{c}\text { Anaplastic } \\
\text { HPC }\end{array}$ & 4 & $1(25.0)$ & & $1(25.0)$ & Grade III & 4 & $1(25.0)$ & & $1(25.0)$ \\
\hline
\end{tabular}
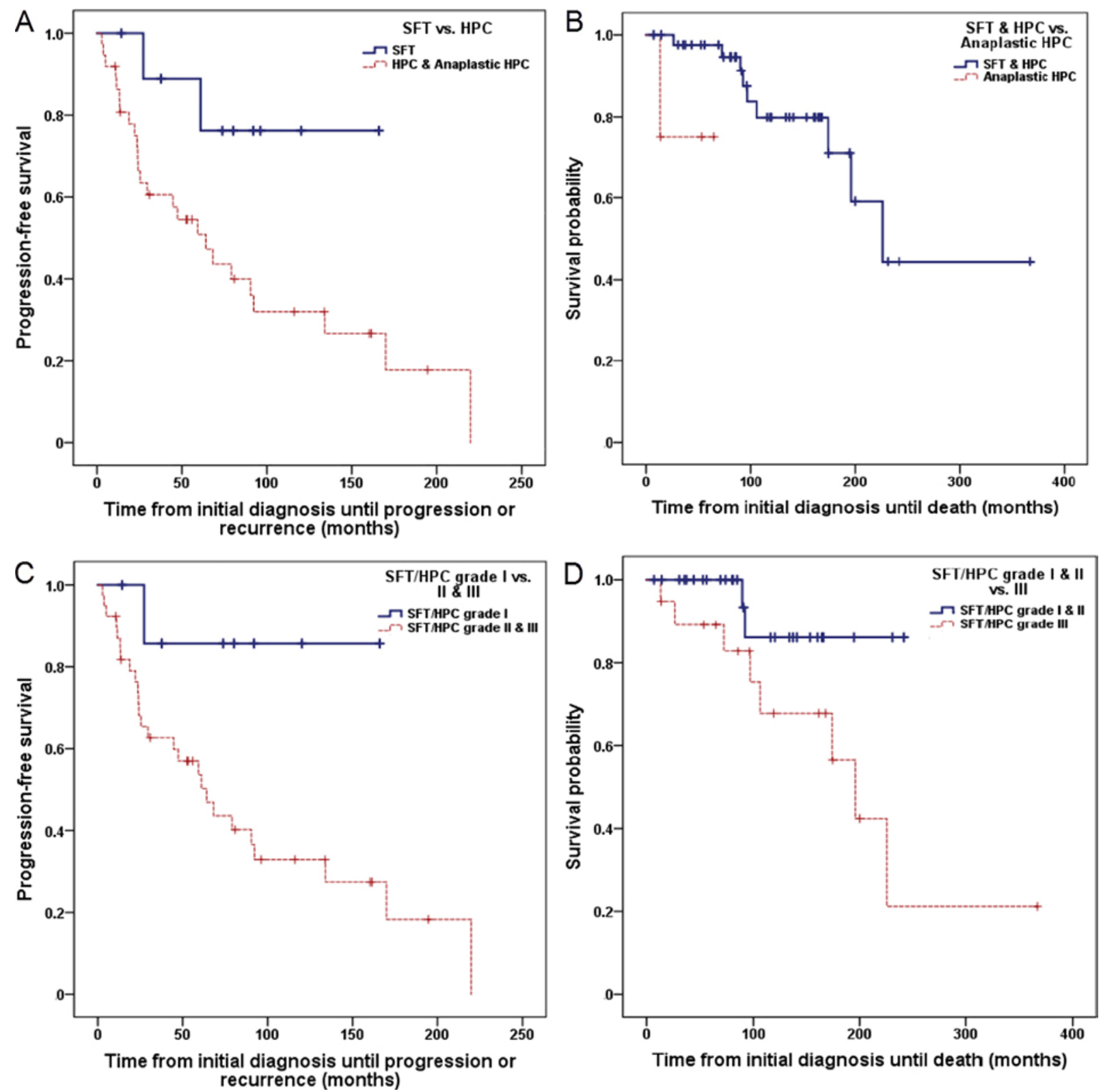

FIG. 4. Kaplan-Meier survival curves showing the pathology-related PFS (A) and OS (B) of patients with SFT and HPC according to the prior (2007) pathological classification. Pathology-related PFS (C) and OS (D) of patients with SFT/HPC according to the 2016 WHO classification. Figure is available in color online only. 

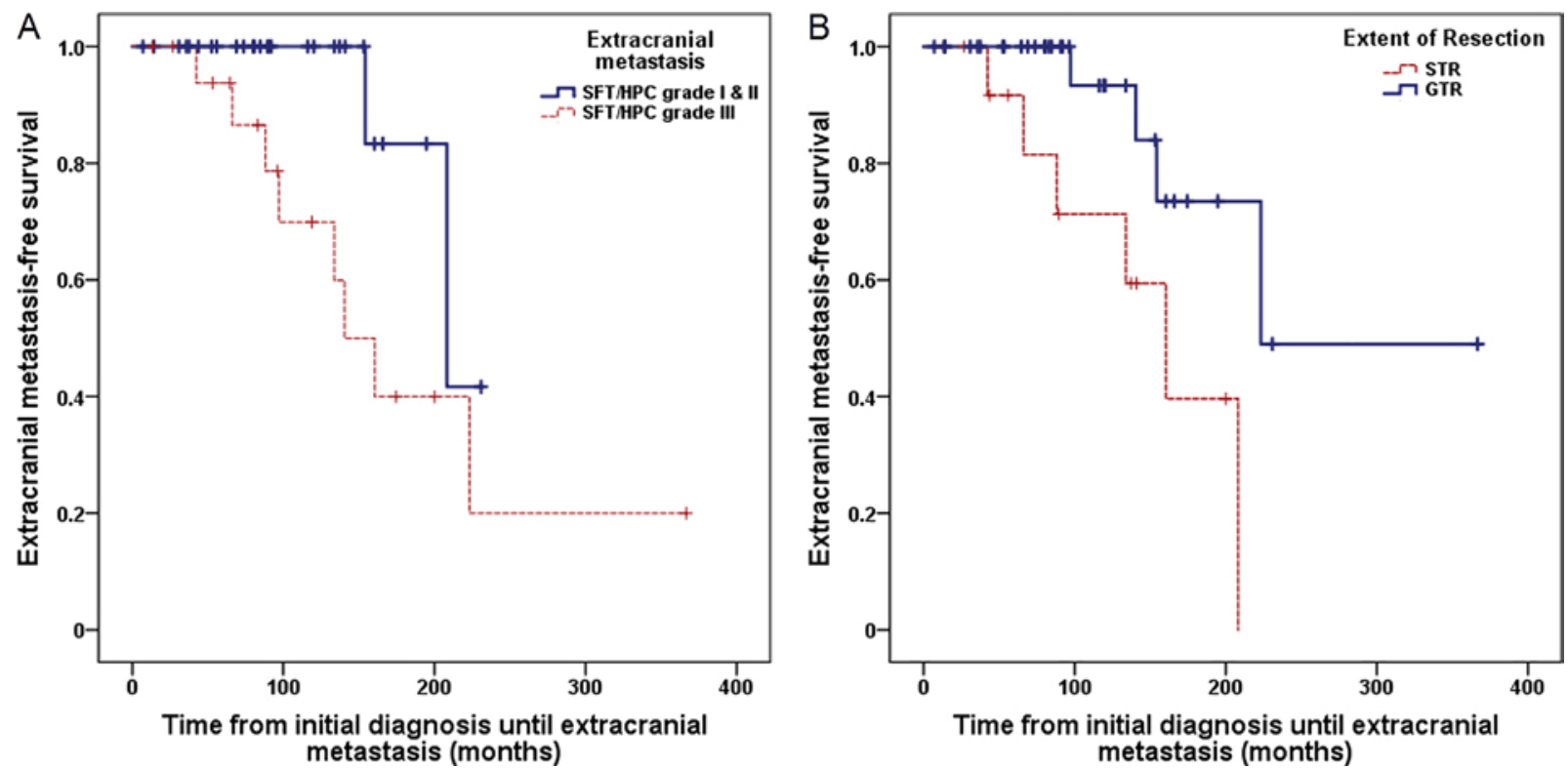

FIG. 5. Kaplan-Meier survival curves showing pathology-related extracranial metastasis-free survival (A) and resection-related extracranial metastasis-free survival (B) of patients with SFT/HPC. Figure is available in color online only.

TABLE 5. Treatment strategy subgroups

\begin{tabular}{|c|c|c|c|c|c|}
\hline Treatment Strategy & Pathology (SFT/HPC) & No. of Patients (\%) & No. of Recurrences (\%) & No. of Extracranial Metastases (\%) & No. of Deaths (\%) \\
\hline \multirow[t]{3}{*}{ GTR alone } & Grade I & $6(12.8)$ & $1(16.7)$ & & $1(16.7)$ \\
\hline & Grade II & $8(17.0)$ & $7(87.5)$ & $1(12.5)$ & \\
\hline & Grade III & $5(10.6)$ & $3(60.0)$ & & \\
\hline \multirow[t]{3}{*}{ GTR + radiation } & Grade I & $2(4.2)$ & & & \\
\hline & Grade II & $6(12.8)$ & & & \\
\hline & Grade III & $6(12.8)$ & $4(66.7)$ & $3(50.0)$ & $2(33.3)$ \\
\hline \multirow[t]{2}{*}{ STR alone } & Grade II & $3(6.4)$ & $3(100)$ & $1(33.3)$ & \\
\hline & Grade III & $6(12.8)$ & $5(83.3)$ & $3(50.0)$ & $5(83.3)$ \\
\hline \multirow[t]{2}{*}{ STR + radiation } & Grade II & $3(6.4)$ & $2(66.7)$ & & $1(33.3)$ \\
\hline & Grade III & $2(4.2)$ & $2(100)$ & $2(100)$ & $1(50.0)$ \\
\hline Total & & $47(100)$ & $27(57.4)$ & $10(21.3)$ & $10(21.3)$ \\
\hline
\end{tabular}

with grade II or III HPC is beneficial for eliminating the risk of recurrence. ${ }^{13,14}$ In the 2013 WHO classification of soft tissue and bone, ${ }^{19}$ the term "HPC" was omitted, leaving the single unified entity of SFT subdivided into levels of malignancy. The 2016 WHO classification of CNS tumors combined intracranial SFT and HPC into a single disease entity because of the discovery of NAB2-STAT6 fusion using whole-exome sequencing.

In our study, median age at the time of diagnosis was 38 years, and males were affected more frequently than females, similar to reports by other investigators..$^{8,28,35}$ When we evaluated prognostic factors associated with PFS and OS by using uni- and multivariate analyses, GTR was an important factor correlated with PFS and OS, and adjuvant radiation therapy was significantly associated with PFS.

Currently, the association of pathological grade with recurrence and survival is controversial. Very few authors have analyzed pathology-related PFS and OS according to the 2016 WHO classification of CNS tumors. Bouvier et al. ${ }^{2}$ classified SFT and HPC into Marseille grades I, IIa, $\mathrm{IIb}$, and III. They reported that the grade of tumor malignancy negatively correlated with patient PFS and OS in the SFT/HPC group. In our study, grading the SFT/ HPC according to the 2016 WHO classification revealed no significant association with PFS and OS ( $\mathrm{p}>0.05)$. However, grade III SFT/HPC cases regraded from conventional SFT or HPC showed higher recurrence rates, more frequent extracranial metastases, and higher mortality rates. These results show that the 2016 WHO classification reflects the malignant pathological potential better than prior pathological classifications. Several studies analyzing the clinical outcome of HPC have reported that high-grade tumors have higher recurrence rates than lowgrade tumors. ${ }^{10,28,35}$ Moreover, Damodaran et al. ${ }^{8}$ demon- 

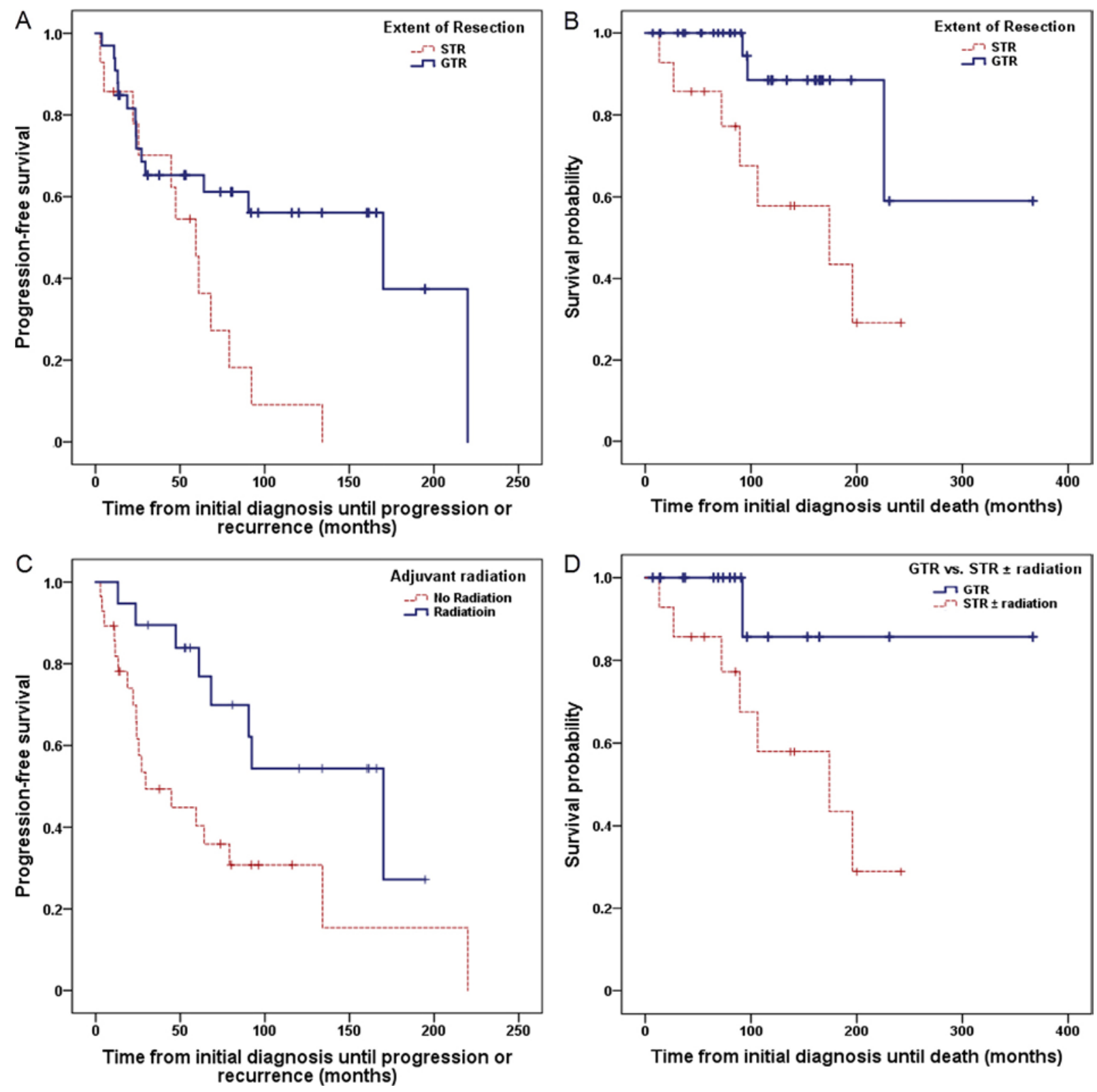

FIG. 6. Kaplan-Meier survival curves showing resection-related PFS (A) and OS (B) and radiation-related PFS (C) and OS (D). Figure is available in color online only.

strated that high-grade tumors were correlated with worse survival rates than low-grade tumors, but there was no significant association between pathological grade and recurrence. Our study showed that grade I SFT/HPC with the classic SFT phenotype led to significantly superior PFS than grade II and III SFT/HPC with an HPC phenotype (146.2 vs 89.7 months, $p=0.043$ ), whereas grade III SFT/HPC led to significantly worse OS than grade I and II SFT/HPC (194.8 vs 220.8 months, $p=0.048$ ). There was also no difference in prognosis for PFS and OS between the prior pathological classifications and the 2016 WHO classification of CNS tumors. Therefore, we concluded that the 2016 WHO classification could better reflect prognosis for PFS and OS than the prior pathologi- cal classifications by unifying the 2 disease entities into the single term "SFT/HPC" and by reflecting the different types of malignant pathological progression. Surely, the 2016 WHO classification does not perfectly reflect all of the possible clinical outcomes of intracranial SFT/HPC. Despite this limitation, our study is the first to analyze the clinical outcome of intracranial SFT/HPC according to the 2016 WHO classification, which reflects the different types of malignant pathological potential better than the prior (2007) classification.

Many studies investigating either SFT or HPC have similarly concluded that GTR is an important prognostic factor for survival and recurrence. ${ }^{4,5,27,31}$ Gross-total resection rates reportedly vary from $20.9 \%$ to $85 \% .^{5,8-10,16,17,21}$, 


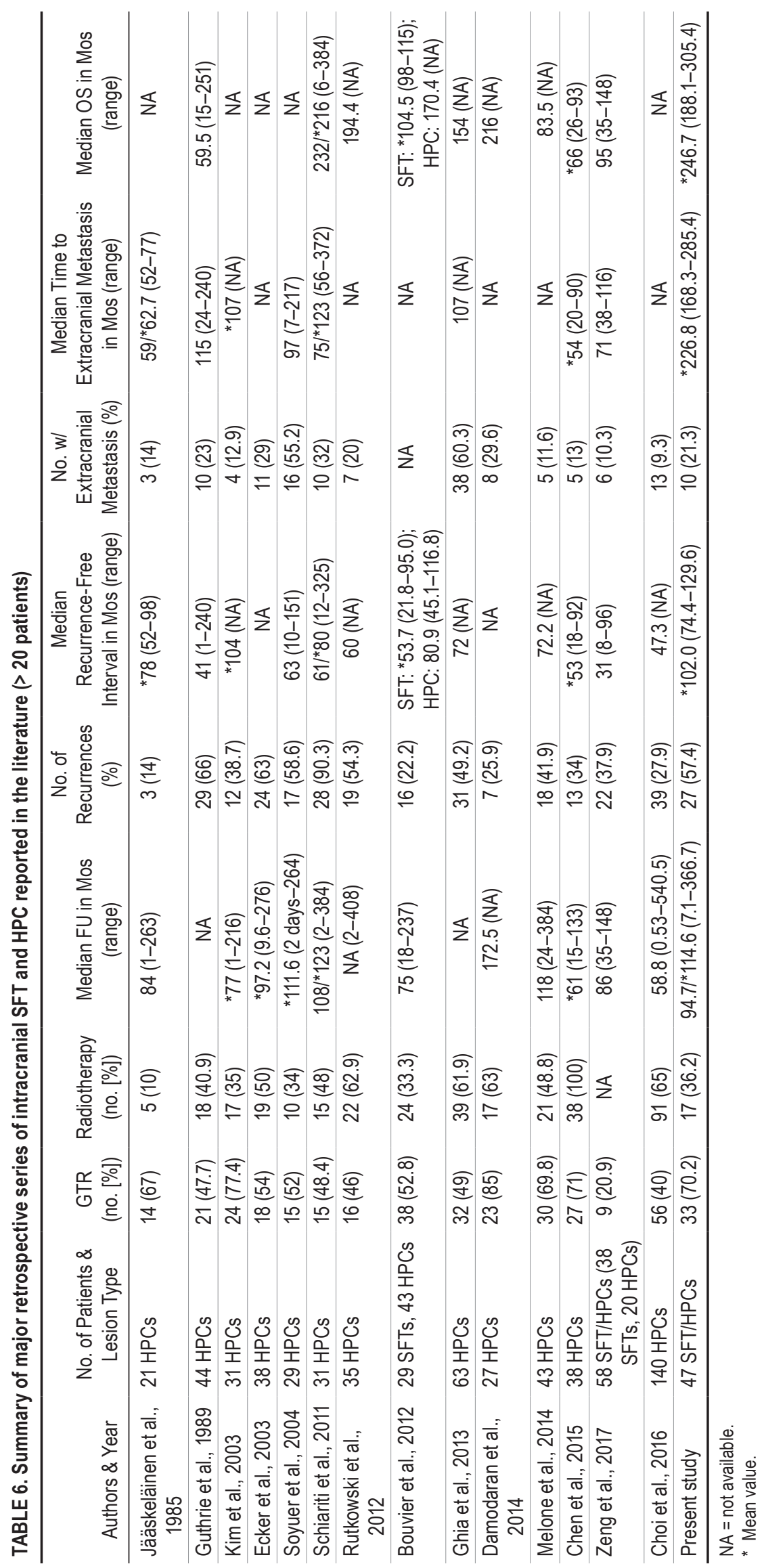


27,33,35,37,43 Several authors have reported that recurrence in intracranial SFT is easier and faster in STR cases than in GTR cases. ${ }^{12,18}$ Chen et al. ${ }^{5}$ reviewed the clinical outcomes of 38 patients with HPC with a mean follow-up of 61 months. In their study, GTR was achieved in $83 \%$ of the cases and the average OS time in the GTR group was significantly higher than that in the STR group (120 vs 68 months, $\mathrm{p}=0.013)$. The mean PFS time in the GTR group was significantly higher than that in the STR group (100 vs 50 months, $p=0.006)$. Melone et al. ${ }^{27}$ described their experience with 43 cases over a median follow-up of 118 months. Gross-total resection was achieved in $70 \%$ of the cases. These authors reported that OS and PFS of the patients treated with GTR were significantly longer than those of the patients treated with STR $(p=0.047$ and 0.0025 , respectively). Meanwhile, Ghia et al. ${ }^{14}$ analyzed 63 patients diagnosed with meningeal HPC and reported a GTR rate of 49\%; in addition, GTR was an independent predictor of improved PFS $(p=0.008)$ but not of OS ( $p$ $=0.54$ ). In our study, the GTR group demonstrated longer OS and PFS than those in the STR group ( $\mathrm{p}<0.05)$, corroborating most other studies. A multidisciplinary approach combining radical resection with adjuvant radiation therapy in any form, including EBRT, stereotactic radiosurgery, and proton beam radiotherapy, was applied in an earlier study; ${ }^{1}$ this strategy was used given concerns regarding metastatic potential and infiltration into nerves, vessels, and neurocritical structures surrounding tumors. Results from analyses of the extent of resection indicated that patients undergoing GTR showed better OS than those undergoing STR with any form of adjuvant radiation. ${ }^{27,33,34}$ In Rutkowski et al.'s review, as in our own study, the patients undergoing GTR alone showed improved OS compared with that in patients undergoing STR with or without adjuvant radiation. ${ }^{34}$ However, Ghia et al. reported that patients who had undergone STR with postoperative EBRT compared favorably in terms of OS with those who had undergone GTR alone. ${ }^{14}$ Therefore, GTR preserving neurological function should be the primary treatment goal for patients with SFT/HPC. Currently, postoperative adjuvant radiation shows contradictory results for survival. Several authors have reported that the use of postoperative adjuvant radiation combined with surgery decreases recurrence rates with no survival benefit, ${ }^{27,33}$ whereas others have reported improved survival and decreased recurrence rates.9,16,35,37,38 Our results showed that the addition of adjuvant radiation regardless of the extent of resection did not significantly improve survival but did reduce recurrence rates $(p=0.018)$. In the literature, a focal fractionated radiotherapy dose of $50 \mathrm{~Gy}$ is recommended as a standard to prevent recurrence..$^{9,13,16,23}$ However, there is no current standard protocol regarding the optimal modality or dose of radiotherapy. There are also few publications in the literature reporting the effects of radiotherapy on SFT. ${ }^{26,32}$ However, we found that there was a significant correlation between adjuvant radiation therapy and PFS in the SFT/HPC group. Therefore, adjuvant radiation therapy can be administered in patients with SFT/HPC that shows malignant features or that has not been completely resected.

The reported incidence of extracranial metastases ranges from $12.0 \%$ to $55.0 \% .^{5,9,21,27,33,35,37,39,40}$ The most common sites of metastasis are the liver, lung, bone, abdominal cavity, kidney, and pancreas. The average time to the development of extracranial metastases is controversial, but the reported interval from the primary operation to the first extracranial metastases has ranged from 8 to 16 years.22 Our study showed similar results for incidence, metastasis sites, and interval to the development of extracranial metastases. Kim et al. reported that extracranial metastases appeared to be more common in high-grade HPC and that the development of extracranial metastases leads to a significant reduction in survival time, with an average of 24 months after the identification of extracranial metastases. ${ }^{20}$ Table 6 summarizes the clinical outcomes in prior studies of SFT and HPC that used either the new or the old grading system. In our assessment, grade III SFT/HPC was strongly correlated with the development of extracranial metastases. Eight of the 10 patients who had died had grade III SFT/HPC, 5 of them had extracranial metastases, and extracranial metastases were confirmed as the direct cause of death in 3 patients. Earlier studies have reported that GTR may favorably affect the development of extracranial metastases, ${ }^{29,43}$ and our data demonstrated that patients treated with GTR had a significantly longer extracranial metastasis-free interval than the patients treated with STR (270.2 vs 149.4 months). Therefore, long-term surveillance for extracranial metastasis is needed to prolong survival among patients who have undergone GTR.

\section{Conclusions}

The 2016 WHO classification of CNS tumors reflects the prognosis for PFS and OS better than prior classifications by unifying 2 disease entities into the single term "SFT/HPC" and by reflecting the different types of malignant pathological progression. Grades II and III SFT/HPC are associated with a reduced time to progression, whereas grade III SFT/HPC is significantly correlated with higher recurrence rates, more frequent extracranial metastases, and higher mortality rates. Gross-total resection preserving neurological function should be the primary treatment goal for SFT/HPC. Radiation can be administered as adjuvant therapy for cases of SFT/HPC that show an aggressive phenotype or that are not treated with GTR. Extracranial metastasis is a common cause of death, particularly in grade III SFT/HPC; therefore, long-term close surveillance is recommended.

\section{References}

1. Bastin KT, Mehta MP: Meningeal hemangiopericytoma: defining the role for radiation therapy. J Neurooncol 14:277287, 1992

2. Bouvier C, Métellus P, de Paula AM, Vasiljevic A, Jouvet A, Guyotat J, et al: Solitary fibrous tumors and hemangiopericytomas of the meninges: overlapping pathological features and common prognostic factors suggest the same spectrum of tumors. Brain Pathol 22:511-521, 2012

3. Carneiro SS, Scheithauer BW, Nascimento AG, Hirose T, Davis DH: Solitary fibrous tumor of the meninges: a lesion distinct from fibrous meningioma. A clinicopathologic and 
immunohistochemical study. Am J Clin Pathol 106:217224, 1996

4. Chen H, Zeng XW, Wu JS, Dou YF, Wang Y, Zhong P, et al: Solitary fibrous tumor of the central nervous system: a clinicopathologic study of 24 cases. Acta Neurochir (Wien) 154:237-248, 2012

5. Chen LF, Yang Y, Yu XG, Gui QP, Xu BN, Zhou DB: Multimodal treatment and management strategies for intracranial hemangiopericytoma. J Clin Neurosci 22:718725,2015

6. Chmielecki J, Crago AM, Rosenberg M, O’Connor R, Walker SR, Ambrogio L, et al: Whole-exome sequencing identifies a recurrent NAB2-STAT6 fusion in solitary fibrous tumors. Nat Genet 45:131-132, 2013

7. Choi J, Park SH, Khang SK, Suh YL, Kim SP, Lee YS, et al: Hemangiopericytomas in the central nervous system: a multicenter study of Korean cases with validation of the usage of STAT6 immunohistochemistry for diagnosis of disease. Ann Surg Oncol 23 (Suppl 5):954-961, 2016

8. Damodaran O, Robbins P, Knuckey N, Bynevelt M, Wong G, Lee G: Primary intracranial haemangiopericytoma: comparison of survival outcomes and metastatic potential in WHO grade II and III variants. J Clin Neurosci 21:13101314,2014

9. Dufour H, Métellus P, Fuentes S, Murracciole X, Régis J, Figarella-Branger D, et al: Meningeal hemangiopericytoma: a retrospective study of 21 patients with special review of postoperative external radiotherapy. Neurosurgery 48:756763, 2001

10. Ecker RD, Marsh WR, Pollock BE, Kurtkaya-Yapicier O, McClelland R, Scheithauer BW, et al: Hemangiopericytoma in the central nervous system: treatment, pathological features, and long-term follow up in 38 patients. J Neurosurg 98:1182-1187, 2003

11. Ellison D, Love S, Carao Chimelli LM, Harding B, Lowe J, Vinter HV, et al: Neuropathology: A Reference Text of CNS Pathology. St. Louis: Mosby Elsevier, 2013

12. Fargen KM, Opalach KJ, Wakefield D, Jacob RP, Yachnis AT, Lister JR: The central nervous system solitary fibrous tumor: a review of clinical, imaging and pathologic findings among all reported cases from 1996 to 2010. Clin Neurol Neurosurg 113:703-710, 2011

13. Ghia AJ, Allen PK, Mahajan A, Penas-Prado M, McCutcheon IE, Brown PD: Intracranial hemangiopericytoma and the role of radiation therapy: a population based analysis. Neurosurgery 72:203-209, 2013

14. Ghia AJ, Chang EL, Allen PK, Mahajan A, Penas-Prado M, McCutcheon IE, et al: Intracranial hemangiopericytoma: patterns of failure and the role of radiation therapy. Neurosurgery 73:624-631, 2013

15. Giannini CRE, Hainfelier JA: Hemangiopericytoma, in Louis DN, Ohgaki H, Wiestler OD, et al (eds): WHO Classification of Tumours of the Central Nervous System, ed 4. Lyon, France: International Agency for Research on Cancer, 2007

16. Guthrie BL, Ebersold MJ, Scheithauer BW, Shaw EG: Meningeal hemangiopericytoma: histopathological features, treatment, and long-term follow-up of 44 cases. Neurosurgery 25:514-522, 1989

17. Jääskeläinen J, Servo A, Haltia M, Wahlström T, Valtonen S: Intracranial hemangiopericytoma: radiology, surgery, radiotherapy, and outcome in 21 patients. Surg Neurol 23:227-236, 1985

18. Jiang N, Xie YY, Chen W, Peng ZF, Yuan XR, Li XJ, et al: solitary fibrous tumor of central nervous system: clinical and prognostic study of 24 cases. World Neurosurg 99:584-592, 2017

19. Jo VY, Fletcher CD: WHO classification of soft tissue tumours: an update based on the 2013 (4th) edition.

Pathology 46:95-104, 2014
20. Kim BS, Kong DS, Seol HJ, Nam DH, Lee JI: Gamma knife radiosurgery for residual or recurrent intracranial hemangiopericytomas. J Clin Neurosci 35:35-41, 2017

21. Kim JH, Jung HW, Kim YS, Kim CJ, Hwang SK, Paek SH, et al: Meningeal hemangiopericytomas: long-term outcome and biological behavior. Surg Neurol 59:47-54, 2003

22. Koyama H, Harada A, Nakao A, Nonami T, Kurokawa T, Kaneko T, et al: Intracranial hemangiopericytoma with metastasis to the pancreas. Case report and literature review. J Clin Gastroenterol 25:706-708, 1997

23. Kumar N, Kumar R, Kapoor R, Ghoshal S, Kumar P, Salunke PS, et al: Intracranial meningeal hemangiopericytoma: 10 years experience of a tertiary care Institute. Acta Neurochir (Wien) 154:1647-1651, 2012

24. Louis DN, Ohgaki H, Wiestler OD, Cavenee WK, Burger PC, Jouvet A, et al: The 2007 WHO classification of tumours of the central nervous system. Acta Neuropathol 114:97-109, 2007

25. Louis DN, Perry A, Reifenberger G, von Deimling A, Figarella-Branger D, Cavenee WK, et al: The 2016 World Health Organization Classification of Tumors of the Central Nervous System: a summary. Acta Neuropathol 131:803820,2016

26. Macfarlane RG, Galloway M, Plowman PN, Thomas DGT: A highly vascular intracranial solitary fibrous tumor treated with radiotherapy and toremifene: case report. Neurosurgery 56:E1378, 2005

27. Melone AG, D'Elia A, Santoro F, Salvati M, Delfini R, Cantore $\mathrm{G}$, et al: Intracranial hemangiopericytoma-our experience in 30 years: a series of 43 cases and review of the literature. World Neurosurg 81:556-562, 2014

28. Mena H, Ribas JL, Pezeshkpour GH, Cowan DN, Parisi JE: Hemangiopericytoma of the central nervous system: a review of 94 cases. Hum Pathol 22:84-91, 1991

29. Morrison DA, Bibby K: Sellar and suprasellar hemangiopericytoma mimicking pituitary adenoma. Arch Ophthalmol 115:1201-1203, 1997

30. Paulus WSB, Perry A: Mesenchymal non-meningothelial tumours, in Louis DN, Ohgaki H, Wiestler OD, et al (eds): WHO Classification of Tumours of the Central Nervous System, ed 4. Lyon, France: International Agency for Research on Cancer, 2007

31. Ramakrishna R, Rostomily R, Sekhar L, Rockhill J, Ferreira M: Hemangiopericytoma: radical resection remains the cornerstone of therapy. J Clin Neurosci 21:612-615, 2014

32. Reames DL, Mohila CA, Sheehan JP: Treatment of intracranial solitary fibrous tumors with gamma knife radiosurgery: report of two cases and review of literature. Neurosurgery 69:E1023-E1028, 2011

33. Rutkowski MJ, Jian BJ, Bloch O, Chen C, Sughrue ME, Tihan T, et al: Intracranial hemangiopericytoma: clinical experience and treatment considerations in a modern series of 40 adult patients. Cancer 118:1628-1636, 2012

34. Rutkowski MJ, Sughrue ME, Kane AJ, Aranda D, Mills SA, Barani IJ, et al: Predictors of mortality following treatment of intracranial hemangiopericytoma. J Neurosurg 113:333339,2010

35. Schiariti M, Goetz P, El-Maghraby H, Tailor J, Kitchen N: Hemangiopericytoma: long-term outcome revisited. Clinical article. J Neurosurg 114:747-755, 2011

36. Schweizer L, Koelsche C, Sahm F, Piro RM, Capper D, Reuss DE, et al: Meningeal hemangiopericytoma and solitary fibrous tumors carry the NAB2-STAT6 fusion and can be diagnosed by nuclear expression of STAT6 protein. Acta Neuropathol 125:651-658, 2013

37. Soyuer S, Chang EL, Selek U, McCutcheon IE, Maor MH: Intracranial meningeal hemangiopericytoma: the role of radiotherapy: report of 29 cases and review of the literature. Cancer 100:1491-1497, 2004 
38. Staples JJ, Robinson RA, Wen BC, Hussey DH: Hemangiopericytoma-the role of radiotherapy. Int J Radiat Oncol Biol Phys 19:445-451, 1990

39. Stout AP, Murray MR: Hemangiopericytoma: a vascular tumor featuring Zimmermann's pericytes. Ann Surg 116:26-33, 1942

40. Sundaram C, Uppin SG, Uppin MS, Rekha JS, Panigrahi MK, Purohit AK, et al: A clinicopathological and immunohistochemical study of central nervous system hemangiopericytomas. J Clin Neurosci 17:469-472, 2010

41. Thway K, Ng W, Noujaim J, Jones RL, Fisher C: The current status of solitary fibrous tumor: diagnostic features, variants, and genetics. Int J Surg Pathol 24:281-292, 2016

42. Tihan T, Viglione M, Rosenblum MK, Olivi A, Burger PC: Solitary fibrous tumors in the central nervous system. A clinicopathologic review of 18 cases and comparison to meningeal hemangiopericytomas. Arch Pathol Lab Med 127:432-439, 2003

43. Wei XE, Wang D, Zhang YZ, Li MH, Li WB: Subtotal resection of cranial hemangiopericytoma accelerates the speed of extracranial metastasis. Neurosurg Q 1:16-18, 2012

44. Zeng L, Wang Y, Wang Y, Han L, Niu H, Zhang M, et al: Analyses of prognosis-related factors of intracranial solitary fibrous tumors and hemangiopericytomas help understand the relationship between the two sorts of tumors. J Neurooncol 131:153-161, 2017

\section{Disclosures}

The authors report no conflict of interest concerning the materials or methods used in this study or the findings specified in this paper.

\section{Author Contributions}

Conception and design: Seol, BS Kim, Suh. Acquisition of data: BS Kim, Y Kim, Suh. Analysis and interpretation of data: BS Kim, Y Kim. Drafting the article: BS Kim. Critically revising the article: Seol, Y Kim, Suh. Reviewed submitted version of manuscript: Seol, Y Kim, Kong, Nam, Lee, Suh. Approved the final version of the manuscript on behalf of all authors: Seol. Statistical analysis: BS Kim. Administrative/technical/material support: Seol, Y Kim, Kong, Nam, Lee. Study supervision: Seol, Suh.

\section{Correspondence}

Ho Jun Seol: Samsung Medical Center, Sungkyunkwan University School of Medicine, Seoul, Republic of Korea. junoseol@ hanmail.net. 